\title{
ICU Management of Traumatic Brain Injury
}

\author{
Matthew R. Hallman • Aaron M. Joffe
}

Published online: 8 February 2013

(c) Springer Science + Business Media New York 2013

\begin{abstract}
Traumatic brain injury (TBI) is a major cause of death and disability throughout the world. Injury can be divided into primary and secondary injuries. For patients with TBI admitted to the intensive care unit (ICU), the management and prevention of secondary injury is most important. The third edition of the Brain Trauma Foundation guidelines was published in 2007 and is widely used to guide treatment of patients with severe TBI. This article reviews ICU care of patients with severe TBI, with a particular focus on recent evidence that is not incorporated in the existing guidelines.
\end{abstract}

Keywords Brain trauma $\cdot$ Neurocritical care .

Traumatic brain injury

\section{Introduction}

Traumatic brain injury (TBI) is a major cause of death and disability throughout the world. In the USA it is estimated that 1.5 to 1.7 million people experience TBI annually $[1,2]$. Globally, the burden is even greater, with 10 million cases estimated each year [3]. Approximately one quarter of these cases are classified as severe.

The Brain Trauma Foundation (BTF) first published guidelines for the management of severe TBI in 1995, and has subsequently revised the guidelines twice, most recently in the third edition published in 2007 [4*0]. The recommendations and management strategies outlined in

M. R. Hallman $(\varangle)$ A A. M. Joffe

Department of Anesthesiology and Pain Medicine, Harborview

Medical Center, University of Washington, 325 Ninth Avenue,

Box 359724, Seattle, WA 98104, USA

e-mail:mhallman@uw.edu these documents represent the foundation of intensive care unit (ICU) management. Nonetheless, they remain largely based on lower-level recommendations owing to a lack of high-quality evidence. The purpose of this review is to highlight recent evidence that addresses the most commonly used interventions for management of severe TBI in the ICU as is relates to the existing BTF guidelines.

\section{Physiology, Pathophysiology, and Principles of Treatment}

Cerebral injury can be broadly categorized as either primary or secondary. Primary injuries are characterized by direct cellular destruction as energy is transferred to the brain. This is commonly seen as the result of gunshot wounds, stabbings, and bone fragments and other projectiles that physically penetrate the skull and destroy normal parenchymal and vascular architecture. Blunt and concussive injuries, although outwardly less obvious, may result in equally destructive levels of energy transfer. Secondary injuries occur not because of the initial energy transfer, but rather as a result of cerebral blood flow (CBF) disruption, inflammatory mediator proliferation, edema, and excitatory neurotransmitter release-all factors that lead to oxygen supply and demand imbalance and ultimately disrupt normal cellular function.

The Fick equation (Eq. 1) describes the relationship between systemic oxygen consumption $\left(\mathrm{VO}_{2}\right)$, cardiac output $(\mathrm{CO})$, the oxygen content of arterial blood $\left(\mathrm{CaO}_{2}\right)$, and the oxygen content of venous blood $\left(\mathrm{CvO}_{2}\right)$ :

$\mathrm{VO}_{2}=\mathrm{CO} \times\left(\mathrm{CaO}_{2}-\mathrm{CvO}_{2}\right)$

An analogous cerebral relationship exists such that the cerebral metabolic rate of oxygen consumption $\left(\mathrm{CMRO}_{2}\right)$ 
can be described by relating $\mathrm{CBF}, \mathrm{CaO}_{2}$, and the oxygen content of jugular venous blood $\left(\mathrm{CjvO}_{2}\right)$ :

$\mathrm{CMRO}_{2}=\mathrm{CBF} \times\left(\mathrm{CaO}_{2}-\mathrm{CjvO}_{2}\right)$

Further, just as Ohm's law (Eq. 3) relates mean arterial pressure (MAP), central venous pressure (CVP), systemic vascular resistance (SVR) and $\mathrm{CO}$, there is again an analogous relationship for the cerebral circulation (Eq. 4) where $\mathrm{CBF}$ and cerebral vascular resistance (CVR) are proportional to cerebral perfusion pressure (CPP), where $\mathrm{CPP}=\mathrm{MAP}-$ intracranial pressure (ICP):

$$
\begin{aligned}
& (\mathrm{MAP}--\mathrm{CVP})=\mathrm{CO} \times \mathrm{SVR} \\
& (\mathrm{MAP}--\mathrm{ICP})=\mathrm{CBF} \times \mathrm{CVR}
\end{aligned}
$$

Therefore, any insult that results in less oxygen being supplied to the brain must be accompanied by a decrease in $\mathrm{CMRO}_{2}$. Conversely, when $\mathrm{CMRO}_{2}$ increases, oxygen delivery must also increase. When cerebral oxygen delivery is insufficient to maintain basic cellular function, infarct ensues. A common clinical scenario is one in which the primary injury results in intracranial hypertension (ICH) and systemic hypotension leading to decreased CPP and brain ischemia. Subsequent respiratory depression results in hypoxemia and exacerbates the primary insult. This causes worsening cerebral ischemia and the release of excitotoxic mediators such as glutamate, and can lead to seizures that further increase cerebral oxygen demand. The resulting downward spiral can worsen ischemia and extend the injured territory. If untreated, this cycle can be fatal. It is with an understanding of these basic principles and with the general goal of minimizing secondary injury by maintaining oxygen supply and minimizing oxygen demand that targets for therapeutic interventions have been developed. Disturbances that should be avoided are listed in Table 1 .

\section{Monitoring of ICP}

\section{ICP, MAP, and CPP Targets}

The optimal therapeutic target for CPP and the upper limit of acceptable ICP remain contentious. Treatment is

Table 1 Determinants of cerebral oxygen supply and demand

\begin{tabular}{ll}
\hline $\begin{array}{l}\text { Factors that decrease } \\
\text { cerebral oxygen supply }\end{array}$ & $\begin{array}{l}\text { Factors that increase } \\
\text { cerebral oxygen demand }\end{array}$ \\
\hline Anemia & Seizure \\
Hypoxemia & Fever \\
Systemic hypotension & Agitation \\
Low cardiac output & Pain \\
Elevated intracranial pressure & Excitatory neurotransmitters \\
Cerebral edema & \\
\hline
\end{tabular}

generally indicated for ICPs greater than $20-25 \mathrm{mmHg}$, and maintenance of a CPP of $50-70 \mathrm{mmHg}$ is recommended [4*0]. Using the current guideline recommendations as treatment thresholds has been reported to improve outcomes [5, 6]. This is highlighted by a recent prospective study reporting a correlation between an ICP of less than $20 \mathrm{mmHg}$ and CPP of more than $60 \mathrm{mmHg}$ with improved Glasgow Outcome Scale scores [6]. Nonetheless, ICPs greater than $20 \mathrm{mmHg}$ may be tolerated without evidence of neurologic deterioration in patients with a normal computerized tomography (CT) scan of the head [7]. In the recently published Decompressive Craniectomy (DECRA) trial [8・•], which compared decompressive craniectomy with medical management in patients with severe TBI and ICP greater than $20 \mathrm{mmHg}$ for $15 \mathrm{~min}$ or more, surgically treated patients had significantly worse scores on the 6-month Extended Glasgow Outcome Scale assessment than those who were medically managed [odds ratio 1.84 , $95 \%$ confidence interval (CI) $1.04-3.24, P=0.003$ ]. The study has been criticized for including an ICP treatment threshold that many consider too low and inconsistent with current clinical practice [9], underscoring the fact that ICP is simply a number and any treatments based upon it must take into careful consideration the potential risks and benefits. Additionally, these results may lend further support to the finding that some individuals tolerate higher ICPs [7].

The critical level of CPP, which is the level below which $\mathrm{CBF}$ is insufficient and cerebral ischemia occurs, is generally felt to be in the range of $50-60 \mathrm{mmHg}$. Additional monitoring techniques, including jugular venous oxygen saturation $\left(\mathrm{SjvO}_{2}\right)$ and brain tissue oxygen tension $\left(\mathrm{PbO}_{2}\right)$ monitoring, may help to further refine critical thresholds in individual patients, but outcome data supporting a specific CPP threshold are generally lacking. A similar physiologic rationale supports the avoidance of systemic hypotension. However, generalizable targets remain difficult to define outside the broad recommendations to maintain a sufficient MAP to keep the CPP in the $50-70-\mathrm{mmHg}$ range at the given ICP. The utility of an accurate ICP assessment is therefore easy to understand.

\section{Whom To Monitor}

Current guidelines recommend invasive ICP monitoring in all TBI patients with a Glasgow Coma Scale score less than 9 after initial resuscitation and an abnormal CT scan of the head [4*0]. An abnormal CT scan of the head is further defined as one exhibiting any of the following: hematoma, contusion, edema, herniation, or compressed basal cisterns. Alternatively, ICP should be monitored in those with a normal CT scan of the head and more than two of the following alternative criteria: age more than 
40 years, systolic blood pressure (SBP) less than $90 \mathrm{mmHg}$, and unilateral or bilateral motor posturing. This recommendation is based largely on an over two decades old retrospective review of 207 patients with severe head injury [10]. Although other studies support the low incidence of ICH in the setting of a normal CT scan of the head $[11,12]$, the larger question is whether knowledge of the ICP is of clinical benefit. Proponents argue that objective data are easier to follow and less prone to bias and misinterpretation than subjective clinical examination findings. Historically this question was difficult to answer directly, owing to the strong physiologic rationale for preventing ICH. The recently published Benchmark Evidence from South American Trials: Treatment of Intracranial Pressure (BEST:TRIP) trial was designed to help answer this question directly by taking advantage of a variation in the practice patterns for the management of severe TBI in South America [13••]. A total of 324 patients with severe TBI were randomly allocated to CT scan of the head and clinical examination with or without the addition of ICP monitoring to guide management. Mortality, ICU length of stay, and functional outcome at 6 months were similar between monitoring strategies. Although the authors are careful to point out that this result does not detract from the importance of managing ICP, it suggests the application of invasive monitoring may be less clear than previously thought.

\section{Invasive ICP Monitoring}

When the decision to monitor ICP is made, consensus on which method to use is lacking. The externalized ventricular drain connected to a strain gauge is commonly used and offers the advantages of allowing therapeutic drainage of cerebral spinal fluid and allowing in vivo calibration. However, drainage of cerebral spinal fluid has not been shown to improve outcomes [14], and there is a risk of infection and hemorrhage [15]. Placement itself may be difficult in patients with small ventricles. Subdural, epidural, subarachnoid, and intraparenchymal monitoring locations have all been studied as potential alternatives to the ventricle. Of these, the intraparenchymally placed microtransducer appears to be the most accurate and consistent $[15,16]$. Although most microtransducer devices lack the ability to be recalibrated in vivo, they show good validity for up to 5 days in vitro, have published complication rates that are generally lower than those for externalized ventricular drains, and may be easier to place. Microtransducers using pneumatic technology, which can be recalibrated in vivo, are also available, but remain untested. At this time, the evidence to recommend one specific monitor over another is lacking.
Noninvasive ICP Monitoring

A variety of methods to measure ICP noninvasively have been studied, including transcranial Doppler ultrasonography $[17,18]$, optic nerve sheath diameter [19-21], fundoscopic papillary examination [22], and tympanic membrane displacement [23]. Unfortunately, all of these techniques are subject to artifact, have been studied in relatively small numbers of patients, require significant expertise to perform, and have only limited correlation with invasively obtained ICPs. Both magnetic resonance imaging [24] and CT [25] have also been studied, but have not been shown to correlate with validated methods.

The recently introduced Neurological Pupil index, which requires an automated pupilometer to assess pupil size and reactivity, is one method which does not require specialized skills to perform or interpret the findings, and may add additional objectivity to the clinical examination [26]. In a study of 134 patients, the degree of pupil reactivity was associated with ICPs, with less reactivity corresponding to higher ICPs. What is more, the pupil changes occurred on average $15.9 \mathrm{~h}$ prior to peak ICP. Although these studies certainly do not provide evidence for avoiding invasive ICP monitoring in all situations, they demonstrate that invasive monitors may not be superior to clinical examination findings in all situations and should be evaluated for risk/benefit on a case-by-case basis.

\section{$\mathrm{PbO}_{2}$ and Metabolism Monitoring}

$\mathrm{PbO}_{2}$ represents the availability of oxygen for cellular oxidative metabolism and, thus the balance between oxygen delivery and consumption. $\mathrm{PbO}_{2}$ monitoring is, therefore, of great interest as it directly measures the local oxygen availability. The current recommendation for a treatment threshold to maintain $\mathrm{PbO}_{2}$ above $20 \mathrm{mmHg}$ is based on a paucity of outcome data, which include the widespread use of historical controls. This is reflected in the strength of the recommendation for their widespread use [27, 28]. More recently a prospectively randomized single-center trial comparing the addition of $\mathrm{PbO}_{2}$ monitoring and targeted treatment to a protocol based on the established guidelines found no difference in mortality or functional outcome [29]. A prospective randomized multicenter phase 2 trial-Brain Tissue Oxygen Monitoring in Traumatic Brain Injury (BOOST-2), ClinicalTrials.gov identifier NCT00974259-comparing the addition of $\mathrm{PbO}_{2}$ monitoring to ICP monitoring versus ICP monitoring alone is currently enrolling patients and may provide further clarity regarding the usefulness of $\mathrm{PbO}_{2}$ monitoring and goal-directed therapy.

Cerebral microdialysis allows further discrimination of the adequacy of oxygen and fuel substrates by directly 
measuring local extracellular brain concentrations of various fuel sources and metabolic products. Nearly any molecule can conceivably be monitored, but glucose, lactate, pyruvate, glutamate, glycerol, and $\mathrm{pH}$ levels are most commonly evaluated. Although there is a growing body of data showing that disturbances in many of these parameters are associated with outcome independent of $\mathrm{PbO}_{2}$ and other factors, whether goal-directed correction of the disturbances can be translated into improved outcomes remains unknown [6, 30-32].

Although ICP monitoring, $\mathrm{CPP}$ monitoring, $\mathrm{PbO}_{2}$ monitoring, and cerebral microdialysis may provide important information, there are limitations common to all techniques. Particularly challenging is locating the monitor in an area of the brain that accurately reflects the conditions in the brain as a whole. Normal anatomic structures such as the falx cerebri and tentorium cerebellum present mechanical barriers to the transmission of pressure from one area of the brain to another such that monitoring on one side may not detect pressure elevations on the other. Hematomas or tumor may similarly limit pressure transduction. Such differences may substantially alter local blood flow and cellular metabolism, which can limit the utility of $\mathrm{PbO}_{2}$ monitoring and cerebral microdialysis. This is highlighted by a recent study reporting that $\mathrm{PbO}_{2}$ values may be significantly higher in normal versus abnormal brain parenchyma [33]. In addition, only $\mathrm{PbO}_{2}$ values from normal brain correlated with outcome. In general, monitoring devices should be located in normal brain tissue considered to be at high risk of secondary injury, although this can often be difficult to achieve. In patients without focal parenchymal injury a right frontal location is recommended $[4 \bullet \bullet$.

\section{$\mathrm{SjvO}_{2}$ Monitoring}

Because focal cerebral oxygen levels are prone to the previously noted limitations, $\mathrm{SjvO}_{2}$ monitoring has been advocated as a better method for assessing global oxygenation of the brain. In a manner analogous to mixed venous oxygen saturation, $\mathrm{SjvO}_{2}$ is theorized to reflect the balance between oxygen supply and demand. Currently, this technique is infrequently employed because of insensitivity to focal ischemia in the face of relatively well preserved global perfusion. However, the sampling of jugular venous blood may still prove useful. The excitatory amino acid glutamate is an excitotoxic mediator associated with poor outcomes and its concentration has been shown to increase rapidly when $\mathrm{SjvO}_{2}$ falls below $50 \%$ [34]. Additional metabolic markers may also be present in jugular vein effluent in sufficient concentrations to be measured, but this requires further study.

\section{Interventions To Improve Cerebral Perfusion}

\section{Systemic Blood Pressure}

Avoiding systemic hypotension is of obvious importance when it comes to ensuring adequate CPP. How this is accomplished is largely outside the scope of this article, but general principles of hemodynamic management in the critically ill apply. Although it is unclear if there is a minimal SBP that should be targeted (MAP, not SBP, is used for calculating CPP), an SBP below $90 \mathrm{mmHg}$ has repeatedly been linked to increased mortality in the TBI population.

\section{Patient Positioning}

Raising the patient's head above the level of the heart by placing the patient in a seated or reverse Trendelenburg position is one of the fastest and least invasive ways to acutely lower ICP $[35,36]$. Although there is commonly concern raised that systemic hypotension will result from this maneuver, it is not associated with negative effects on CPP [14] and is considered a standard part of ventilator-associated pneumonia prophylaxis in mechanically ventilated patients, thus making it a recommended first-line intervention.

\section{Hyperventilation}

Hyperventilation (acute hypocapnea) causes cerebral vasoconstriction by increasing $\mathrm{pH}$ and therefore decreasing the volume of blood in the head. Conversely, hypoventilation decreases $\mathrm{pH}$, which leads to cerebral vasodilatation and increased cerebral blood volume. Although changes in $\mathrm{PaCO}_{2}$ may acutely change ICP for short periods, evidence suggests that the reduction in $\mathrm{CBF}$ associated with prolonged hyperventilation to a $\mathrm{PaCO}_{2}$ below $34 \mathrm{mmHg}$ may be deleterious [14]. In addition, physiologic compensatory mechanisms to ongoing hyperventilation return the systemic $\mathrm{pH}$ to normal within $6-8 \mathrm{~h}$, and a rebound acute respiratory acidosis with the associated cerebral vasodilatation and increased ICP may occur upon return to normoventilation [32]. Hyperventilation is, therefore, only recommended as a short-duration temporizing measure, and hypoventilation should be aggressively avoided.

\section{Osmotherapy}

Numerous hyperosmolar agents have been studied for short-term and prolonged reduction of ICP, but mannitol and hypertonic saline are by far the most commonly used agents clinically. Both are thought to work by two distinct mechanisms. First, plasma volume expansion and dehydration of erythrocytes may improve blood viscosity. 
Second, as a result of establishment of an osmolar gradient where the serum is hyperosmolar relative to brain tissue, water moves out of brain tissue, thereby reducing cerebral edema. A 2003 Cochrane review found $20 \%$ mannitol was safe and effective in lowering ICP in patients with intracranial hemorrhage and planned craniotomy [37]. Two studies analyzed in the review compared high-dose $(1.2-1.4 \mathrm{~g} / \mathrm{kg})$ versus low-dose $(0.6-0.7 \mathrm{~g} / \mathrm{kg})$ rapid infusion of mannitol and found the high-dose regimen to be superior [relative risk (RR) of death $0.56,95 \%$ CI $0.39-0.79]$. The use of $0.25-1 \mathrm{~g} / \mathrm{kg}$ is recommended by the BTF guidelines [4*0]. A subsequent Cochrane review performed in 2007 [38] confirmed the results of the previous Cochrane review, and also found mannitol to be superior to barbiturates but inferior to hypertonic sodium lactate with regard to mortality. However, the $95 \%$ CI for all comparisons except for high-dose versus low-dose mannitol crossed zero.

Hypertonic saline solutions are also commonly used and may even be superior to mannitol. Multiple studies comparing mannitol and hypertonic saline have shown a consistently increased improvement in ICH in the hypertonic saline arms, but hypertonic saline may also be associated with increases in other interventions such as the need for more frequent serum sodium monitoring, and a mortality and functional outcome difference is not noted in these individual trials [39-41]. However, two recent meta-analyses both found that combining the effect sizes from individual trials reveals consistently more effective ICP lowering in the hypertonic saline group compared with the mannitol group (RR of effective ICP control 1.16, $95 \%$ CI 1.00-1.33) [42•, 43]. The ICP lowering effect of hypertonic saline appears consistent over a wide range of concentrations from 3 to $23.4 \%$ [25, 30, 40, 41, 42•, 43-45]. Most often the clinical choice of mannitol or hypertonic saline, and if hypertonic saline, which concentration is chosen, is guided by patient-specific factors such the starting serum sodium level, presumed intravascular volume status, and whether or not central venous access is present as hypertonic saline often requires this, whereas mannitol can be administered peripherally. Whether bolus dosing or continuous infusions are more effective remains unclear as the heterogeneity of the trials in the most recent meta-analysis precludes this determination [43]. Serum osmolarity should be monitored with any hyperosmolar therapy, as serum osmole loads greater than 320-330 mOsm may be harmful, and serum sodium level should generally not be allowed to go above $160 \mathrm{mEq} / \mathrm{L}$ [46].

\section{Hypothermia}

On the basis of a systematic review of 12 trials involving more than 1,300 patients, a benefit from mild to moderate
(32-34 ${ }^{\circ} \mathrm{C}$ ) induced hypothermia was reported compared with simply maintaining normothermia (RR of mortality $0.73,95 \%$ CI $0.62-0.85$; RR of good neurologic outcome 1.52, $95 \%$ CI 1.28-1.80) [47]. However, the beneficial effects of cooling were only found for patients cooled for more than $48 \mathrm{~h}$. In contrast, the more recent National Acute Brain Injury Study: Hypothermia II (NABIS: H2), which enrolled more than 200 patients, failed to find a benefit for cooling [48*•]. The European Study of Therapeutic Hypothermia $\left(32-35{ }^{\circ} \mathrm{C}\right)$ for ICP Reduction After Traumatic Brain Injury (EUROTHERM3235) trial, a prospective randomized multicenter trial with a target enrollment of over 1,300 patients, is currently ongoing, with an anticipated completion sometime in 2013. On the basis of current data and pending further evidence, it would be reasonable to target mild hypothermia in severe TBI patients. On the other hand, avoidance of hyperthermia and aggressive treatment of fever is strongly recommended as pyrexia is highly associated with worse outcomes [4••].

\section{Seizure Prophylaxis}

Seizures have been reported in up to $50 \%$ of patients with TBI and have potentially deleterious effects on neurophysiology [4*0]. Current guidelines recommend routine antiepileptic prophylaxis not be continued for longer than 7 days from the time of injury unless seizure activity is present. Multiple comparisons of leviteracetam with phenytoin have failed to show superiority of one drug over the other, results which have been confirmed in a recent metaanalysis [49].

\section{Sedation in the Mechanically Ventilated}

Tracheal intubation and mechanical ventilation are often components of early resuscitation in severe TBI. Pain and agitation, either as a consequence of the initial injury or caused by the presence of the ventilator, should be treated in order to avoid potential increases in cerebral oxygen requirements. Although a comprehensive review of sedation and analgesia in the critically ill patient is beyond the scope of this article, it is strongly recommended that patients be assessed for pain, agitation, and delirium on a routine basis using standardized and validated assessment tools [50]. A point of contention is whether sedation, whatever agents are used, should be continuously titrated to a particular depth or whether daily interruptions should take place as has been recommended for other populations of critically ill patients. In one observational study of 127 neurologic wake-up trials among 21 severely brain injured adults, the mean ICPs and CPPs modestly increased when continuous propofol infusions were interrupted [51]. In the 
TBI patients $(n=12)$, the ICP increased from $13.4 \pm 6 \mathrm{mmHg}$ at the baseline to $22.7 \pm 12 \mathrm{mmHg}$ $(P<0.05)$ and the CPP increased from $75.6 \pm 11$ to $79.1 \pm 21 \mathrm{mmHg}(P<0.05)$. It was concluded that since most of the patients experienced mild and transient elevations in ICP/CPP, repeated-wake-up trials are not precluded in these patients. In contrast, another prospective observational trial recently reported that among 54 sedative-interruption trials performed on 20 patients over 82 study days, one third of the trials required cessation owing to ICP crisis (ICP greater than $20 \mathrm{mmHg}$ ), agitation, or oxygen desaturation [52]. Critically low levels of $\mathrm{PbO}_{2}$ (less than $20 \mathrm{mmHg}$ ) were observed in $67 \%$ of the aborted trials. Although there has been some historical apprehension to use sedative and analgesic agents because of their tendency to obscure the neurological examination, the most recent TBI guidelines [4••] as well as general critical care guidelines [50] recommend their use as a routine method of improving patient comfort when anxiety and pain are present. They should be titrated to the minimum dosage required to achieve a satisfactory effect in all critically ill patients, including those with TBI.

\section{Burst Suppression}

Although numerous agents, including benzodiazepines [53], propofol [53, 54], barbiturates [40], dexmedetomidine [55], and opioids [56], have been studied and clinically implemented to directly or indirectly lower cerebral oxygen consumption, $\mathrm{CBF}$, and ICP, these effects have not been shown to improve outcomes in rigorous trials and meta-analyses [57•]. Of these agents, only propofol and barbiturates can readily achieve electroencephalographic burst suppression when used alone, although ketamine [58] and dexmedetomidine [59] may reduce the dose of thiopental or propofol required when used in combination. Unfortunately, the use of propofol and barbiturates in doses sufficient to achieve burst suppression is associated with a $25 \%$ incidence of systemic hypotension [57•]. Barbiturates have accrued the most extensive clinical record, with at least one head-to-head trial finding thiopental to be more effective at ICP lowering than pentobarbital. However, this was a small study, did not show an outcome benefit, and there were some differences in baseline characteristics between groups [60]. Propofol may be considered for short-duration applications, but concern for the propofolrelated infusion syndrome often precludes the high doses necessary for burst suppression in the ICU. Despite the traditional teaching that ketamine increases ICP, a substantial body of evidence is conflicting regarding its effects on cerebral physiology [58, 59, 61]. Regardless of the agent chosen, it is recommended that the electroencephalogram be monitored continuously in order to assess burst suppression and minimize sedative doses.

\section{Decompressive Craniectomy}

Surgical management may be necessary when ICP is refractory to all medical interventions or when the initial presentation includes a pending herniation syndrome where waiting for noninvasive measures to be effective is not considered reasonable. Although prompt relief of $\mathrm{ICH}$ has been consistently documented, evidence for improved overall outcome is lacking [62], In fact, The Decompressive Craniectomy in Diffuse TBI (DECRA) trial reported a higher mortality in patients randomized to undergo surgical decompression compared with those randomized to undergo continued medical management when ICPs remained greater than $20 \mathrm{mmHg}$ for $15 \mathrm{~min}$ [8]. However, as mentioned earlier, the threshold may have been set too low to make this a clinically relevant study, and debate regarding the usefulness of the procedure continues [63]. The Randomized Evaluation of Surgery with Craniectomy for Uncontrollable Elevation of ICP (RESCUE-ICP) trial is an ongoing multicenter, multinational prospective controlled trial designed to further define the role of surgical decompression in patients with medically refractory $\mathrm{ICH}$ [64]. Criteria for randomization include ICP greater than $25 \mathrm{mmHg}$ for more than $1 \mathrm{~h}$. As the inclusion criteria are more in line with current clinical practice, the results will likely be more generalizable. Until more robust data become available, decompressive craniectomy for patients with medically refractory ICP remains a reasonable option.

\section{Conclusion}

Although there remains little high-quality evidence on which to base strong recommendations for critical care treatment of patients with severe TBI, extensive clinical experience is available on which to inform clinical decisions. The BTF guidelines remain a cornerstone. However, in light of recently published trial results, highlighted herein, some modifications to the existing recommendations may be considered. A suggested clinical treatment escalation pathway is presented in Fig. 1.

Disclosure Matthew R. Hallman: none; Aaron M. Joffe: none.

\section{References}

Papers of particular interest, published recently, have been highlighted as: 

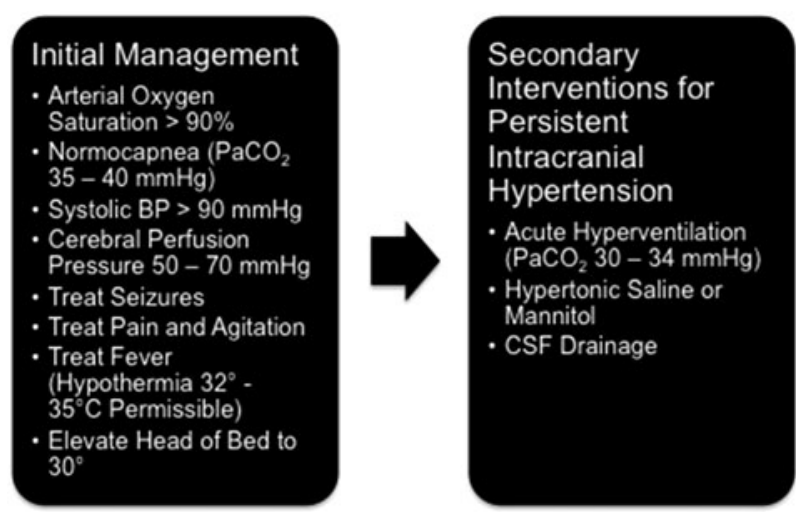

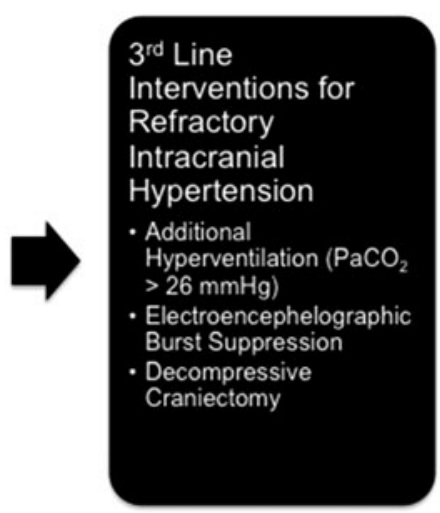

Fig. 1 Intracranial hypertension (ICH) is defined as intracranial pressure greater than $20-25 \mathrm{mmHg}$. Persistent $\mathrm{ICH}$ is defined as $\mathrm{ICH}$ for more than 15-20 min. Refractory ICH is defined as ICH lasting more than $1 \mathrm{~h}$ despite interventions. Hyperventilation should only be

- Of importance

- Of major importance

1. Faul M XL, Wald M, Coronado V. Traumatic brain injury in the United States: emergency department visits, hospitalizations and deaths 2002-2006. Atlanta: Centers for Disease Control and Prevention; 2010.

2. Sosin DM, Sniezek JE, Waxweiler RJ. Trends in death associated with traumatic brain injury, 1979 through 1992. Success and failure. JAMA. 1992;1995(273):1778-80.

3. Hyder AA, Wunderlich CA, Puvanachandra P, Gururaj G, Kobusingye OC. The impact of traumatic brain injuries: a global perspective. Neurorehabilitation. 2007;22:341-53.

4. - Bullock R, et al. Guidelines for the management of severe traumatic brain injury. J Neurotrauma. 2007. This is the most recent edition of the BTF guidelines for the treatment of severe TBI. The guidelines are the cornerstone of contemporary ICU management of TBI.

5. Arabi YM, Haddad S, Tamim HM, et al. Mortality reduction after implementing a clinical practice guidelines-based management protocol for severe traumatic brain injury. J Crit Care. 2010; 25:190-5.

6. Nelson DW, Thornquist B, MacCallum RM, et al. Analyses of cerebral microdialysis in patients with traumatic brain injury: relations to intracranial pressure, cerebral perfusion pressure and catheter placement. BMC Med. 2011;9:21.

7. Chambers IR, Treadwell L, Mendelow AD. Determination of threshold levels of cerebral perfusion pressure and intracranial pressure in severe head injury by using receiver-operating characteristic curves: an observational study in 291 patients. J Neurosurg. 2001;94:412-6.

8. • Cooper DJ, Rosenfeld JV, Murray L, et al. Decompressive craniectomy in diffuse traumatic brain injury. $\mathrm{N}$ Engl J Med. 2011;364:1493-502. This is a prospective, randomized clinical trial comparing medical versus surgical management of patients with severe TBI and refractory ICH. Increased mortality was demonstrated in the surgical treatment group. This study has sparked considerable editorial debate over the generalizability of the criteria for surgical intervention.

9. Honeybul S, Ho KM, Lind CR. What can be learned from the DECRA Study. World Neurosurg. 2012. doi:10.1016/j.wneu. 2012.08.012. used as an short-duration temporizing measure, and should not be maintained for more than 15-20 min. Decompressive craniectomy may be considered at any time when a herniation syndrome is felt to be impending
10. Narayan RK, Kishore PR, Becker DP, et al. Intracranial pressure: to monitor or not to monitor? A review of our experience with severe head injury. J Neurosurg. 1982;56:650-9.

11. Poca MA, Sahuquillo J, Baguena M, Pedraza S, Gracia RM, Rubio E. Incidence of intracranial hypertension after severe head injury: a prospective study using the Traumatic Coma Data Bank classification. Acta Neurochir Suppl. 1998;71:27-30.

12. Lobato RD, Sarabia R, Rivas JJ, et al. Normal computerized tomography scans in severe head injury. Prognostic and clinical management implications. J Neurosurg. 1986;65:784-9.

13. • Chesnut RM, Temkin N, Carney N, et al. A trial of intracranial-pressure monitoring in traumatic brain injury. N Engl J Med 2012;367:2471-81. This prospective, randomized clinical trial comparing invasive versus noninvasive ICP monitoring in patients with severe TBI challenges the convention that invasive ICP monitoring is always necessary. No differences in mortality or functional outcomes were found between groups. There are important differences in health care delivery systems between the study population and fully developed countries that may limit generalizability, but the results are intriguing.

14. Meyer MJ, Megyesi J, Meythaler J, et al. Acute management of acquired brain injury part I: an evidence-based review of nonpharmacological interventions. Brain Inj. 2010;24:694-705.

15. Raboel PH, Bartek J Jr, Andresen M, Bellander BM, Romner B. Intracranial pressure monitoring: invasive versus non-invasive methods-a review. Crit Care Res Pract. 2012;2012:950393.

16. Smith M. Monitoring intracranial pressure in traumatic brain injury. Anesth Analg. 2008;106:240-8.

17. Behrens A, Lenfeldt N, Ambarki K, Malm J, Eklund A, Koskinen LO. Transcranial Doppler pulsatility index: not an accurate method to assess intracranial pressure. Neurosurgery. 2010;66:1050-7.

18. Brandi G, Bechir M, Sailer S, Haberthur C, Stocker R, Stover JF. Transcranial color-coded duplex sonography allows to assess cerebral perfusion pressure noninvasively following severe traumatic brain injury. Acta Neurochir. 2010;152:965-72.

19. Soldatos T, Karakitsos D, Chatzimichail K, Papathanasiou M, Gouliamos A, Karabinis A. Optic nerve sonography in the diagnostic evaluation of adult brain injury. Crit Care. 2008;12:R67.

20. Rajajee V, Vanaman M, Fletcher JJ, Jacobs TL. Optic nerve ultrasound for the detection of raised intracranial pressure. Neurocrit Care. 2011;15:506-15

21. Geeraerts T, Launey Y, Martin L, et al. Ultrasonography of the optic nerve sheath may be useful for detecting raised intracranial 
pressure after severe brain injury. Intensive Care Med. 2007;33: 1704-11.

22. Sinclair AJ, Burdon MA, Nightingale PG, et al. Rating papilloedema: an evaluation of the Frisen classification in idiopathic intracranial hypertension. J Neurol. 2012;259:1406-12.

23. Shimbles S, Dodd C, Banister K, Mendelow AD, Chambers IR. Clinical comparison of tympanic membrane displacement with invasive intracranial pressure measurements. Physiol Meas. 2005;26:1085-92.

24. Marshall I, MacCormick I, Sellar R, Whittle I. Assessment of factors affecting MRI measurement of intracranial volume changes and elastance index. Br J Neurosurg. 2008;22:389-97.

25. Hiler M, Czosnyka M, Hutchinson P, et al. Predictive value of initial computerized tomography scan, intracranial pressure, and state of autoregulation in patients with traumatic brain injury. J Neurosurg. 2006;104:731-7.

26. Chen JW, Gombart ZJ, Rogers S, Gardiner SK, Cecil S, Bullock RM. Pupillary reactivity as an early indicator of increased intracranial pressure: the introduction of the Neurological Pupil index. Surg Neurol Int. 2011;2:82.

27. Spiotta AM, Stiefel MF, Gracias VH, et al. Brain tissue oxygendirected management and outcome in patients with severe traumatic brain injury. J Neurosurg. 2010;113:571-80.

28. Narotam PK, Morrison JF, Nathoo N. Brain tissue oxygen monitoring in traumatic brain injury and major trauma: outcome analysis of a brain tissue oxygen-directed therapy. J Neurosurg. 2009;111:672-82.

29. Green JA, Pellegrini DC, Vanderkolk WE, Figueroa BE, Eriksson EA. Goal directed brain tissue oxygen monitoring versus conventional management in traumatic brain injury: an analysis of in hospital recovery. Neurocrit Care. 2012. doi:10.1007/s12028012-9797-7.

30. Timofeev I, Carpenter KL, Nortje J, et al. Cerebral extracellular chemistry and outcome following traumatic brain injury: a microdialysis study of 223 patients. Brain. 2011;134:484-94.

31. Hillered L, Persson L, Nilsson P, Ronne-Engstrom E, Enblad P. Continuous monitoring of cerebral metabolism in traumatic brain injury: a focus on cerebral microdialysis. Curr Opin Crit Care. 2006;12:112-8.

32. Paraforou T, Paterakis K, Fountas K, et al. Cerebral perfusion pressure, microdialysis biochemistry and clinical outcome in patients with traumatic brain injury. BMC Res Notes. 2011;4: 540.

33. Ponce LL, Pillai S, Cruz J, et al. Position of probe determines prognostic information of brain tissue $\mathrm{PO}_{2}$ in severe traumatic brain injury. Neurosurgery. 2012;70:1492-502; discussion 1502-3.

34. Hlatky R, Furuya Y, Valadka AB, Goodman JC, Robertson CS. Comparison of microdialysate arginine and glutamate levels in severely head-injured patient. Acta Neurochir Suppl. 2002;81: 347-9.

35. Ng I, Lim J, Wong HB. Effects of head posture on cerebral hemodynamics: its influences on intracranial pressure, cerebral perfusion pressure, and cerebral oxygenation. Neurosurgery. 2004;54:593-7; discussion 598.

36. Schulz-Stubner S, Thiex R. Raising the head-of-bed by 30 degrees reduces ICP and improves CPP without compromising cardiac output in euvolemic patients with traumatic brain injury and subarachnoid haemorrhage: a practice audit. Eur J Anaesthesiol. 2006;23:177-80.

37. Roberts I, Schierhout G, Wakai A. Mannitol for acute traumatic brain injury. Cochrane Database Syst Rev. 2003:CD001049.

38. Wakai A, Roberts I, Schierhout G. Mannitol for acute traumatic brain injury. Cochrane Database Syst Rev. 2007:CD001049.

39. Vialet R, Albanese J, Thomachot L, et al. Isovolume hypertonic solutes (sodium chloride or mannitol) in the treatment of refractory posttraumatic intracranial hypertension: $2 \mathrm{~mL} / \mathrm{kg} 7.5 \%$ saline is more effective than $2 \mathrm{~mL} / \mathrm{kg} 20 \%$ mannitol. Crit Care Med. 2003;31:1683-7.

40. Battison C, Andrews PJ, Graham C, Petty T. Randomized, controlled trial on the effect of a $20 \%$ mannitol solution and a $7.5 \%$ saline $/ 6 \%$ dextran solution on increased intracranial pressure after brain injury. Crit Care Med. 2005;33:196-202; discussion 257-8.

41. Kerwin AJ, Schinco MA, Tepas JJ III, Renfro WH, Vitarbo EA, Muehlberger M. The use of $23.4 \%$ hypertonic saline for the management of elevated intracranial pressure in patients with severe traumatic brain injury: a pilot study. J Trauma. 2009;67:277-82.

42. - Kamel H, Navi BB, Nakagawa K, Hemphill JC, III, Ko NU. Hypertonic saline versus mannitol for the treatment of elevated intracranial pressure: a meta-analysis of randomized clinical trials. Crit Care Med. 2011;39:554-9. This is a well-conducted meta-analysis of trials comparing the ICP lowering efficacy of hypertonic saline with that of mannitol. Although the effectiveness was found to be superior with hypertonic saline, the analysis is remarkable for the relatively small number of subjects included. The results have been cited as evidence in favor of the superiority of hypertonic saline as a first-line therapy for elevated ICP by some, but others have interpreted the study as insufficient to change practice.

43. Mortazavi MM, Romeo AK, Deep A, et al. Hypertonic saline for treating raised intracranial pressure: literature review with metaanalysis. J Neurosurg. 2012;116:210-21.

44. Ware ML, Nemani VM, Meeker M, Lee C, Morabito DJ, Manley GT. Effects of $23.4 \%$ sodium chloride solution in reducing intracranial pressure in patients with traumatic brain injury: a preliminary study. Neurosurgery. 2005;57:727-36; discussion 736.

45. Sakellaridis N, Pavlou E, Karatzas S, et al. Comparison of mannitol and hypertonic saline in the treatment of severe brain injuries. J Neurosurg. 2011;114:545-8.

46. Marshall GT, James RF, Landman MP, et al. Pentobarbital coma for refractory intra-cranial hypertension after severe traumatic brain injury: mortality predictions and one-year outcomes in 55 patients. J Trauma. 2010;69:275-83.

47. Fox JL, Vu EN, Doyle-Waters M, Brubacher JR, Abu-Laban R, $\mathrm{Hu}$ Z. Prophylactic hypothermia for traumatic brain injury: a quantitative systematic review. CJEM. 2010;12:355-64.

48. • Clifton GL, Valadka A, Zygun D, et al. Very early hypothermia induction in patients with severe brain injury (the National Acute Brain Injury Study: Hypothermia II): a randomised trial. Lancet Neurol. 2011;10:131-9. This is a prospective, randomized trial in severe TBI patients comparing the effect of induced hypothermia to $33{ }^{\circ} \mathrm{C}$ versus normothermia on 6-month outcomes. The trial was stopped early for futility and no difference was noted. Maintenance of hypothermia for only $48 \mathrm{~h}$, a duration potentially insufficient to impart benefit, has been postulated as the factor responsible for the lack of benefit. The design and limitations of this trial have greatly informed the design and conduct of the ongoing EUROTHERM3235 trial.

49. Zafar SN, Khan AA, Ghauri AA, Shamim MS. Phenytoin versus leviteracetam for seizure prophylaxis after brain injury-a meta analysis. BMC Neurol. 2012;12:30.

50. Barr J, Fraser GL, Puntillo K, et al. Clinical practice guidelines for the management of pain, agitation, and delirium in adult patients in the intensive care unit. Crit Care Med. 2013;41:278-80.

51. Skoglund K, Enblad P, Marklund N. Effects of the neurological wake-up test on intracranial pressure and cerebral perfusion pressure in brain-injured patients. Neurocrit Care. 2009;11: 135-42.

52. Helbok R, Kurtz P, Schmidt MJ, et al. Effects of the neurological wake-up test on clinical examination, intracranial pressure, brain metabolism and brain tissue oxygenation in severely braininjured patients. Crit Care. 2012;16:R226. 
53. Sanchez-Izquierdo-Riera JA, Caballero-Cubedo RE, Perez-Vela JL, Ambros-Checa A, Cantalapiedra-Santiago JA, Alted-Lopez E. Propofol versus midazolam: safety and efficacy for sedating the severe trauma patient. Anesth Analg. 1998;86:1219-24.

54. Kelly DF, Goodale DB, Williams J, et al. Propofol in the treatment of moderate and severe head injury: a randomized, prospective double-blinded pilot trial. J Neurosurg. 1999;90:1042-52.

55. James ML, Olson DM, Graffagnino C. A pilot study of cerebral and haemodynamic physiological changes during sedation with dexmedetomidine or propofol in patients with acute brain injury. Anaesth Intensive Care. 2012;40:949-57.

56. Meyer MJ, Megyesi J, Meythaler J, et al. Acute management of acquired brain injury part II: an evidence-based review of pharmacological interventions. Brain Inj. 2010;24:706-21.

57. - Roberts I, Sydenham E. Barbiturates for acute traumatic brain injury. Cochrane Database Syst Rev. 2012;12:CD000033. This Cochrane review examined the effect of barbiturate use, predominantly to induce electroencephalographic burst suppression in patients with severe TBI, on mortality. Although no effect on mortality was found, the increased incidence of side effects, including a $25 \%$ incidence of hypotension in the barbiturate group, provides compelling evidence to routinely avoid use of barbiturates.
58. Albanese J, Arnaud S, Rey M, Thomachot L, Alliez B, Martin C. Ketamine decreases intracranial pressure and electroencephalographic activity in traumatic brain injury patients during propofol sedation. Anesthesiology. 1997;87:1328-34.

59. Buhrer M, Mappes A, Lauber R, Stanski DR, Maitre PO. Dexmedetomidine decreases thiopental dose requirement and alters distribution pharmacokinetics. Anesthesiology. 1994;80:1216-27.

60. Perez-Barcena J, Llompart-Pou JA, Homar J, et al. Pentobarbital versus thiopental in the treatment of refractory intracranial hypertension in patients with traumatic brain injury: a randomized controlled trial. Crit Care. 2008;12:R112.

61. Flower O, Hellings S. Sedation in traumatic brain injury. Emerg Med Int. 2012;2012:637171.

62. Sahuquillo J, Arikan F. Decompressive craniectomy for the treatment of refractory high intracranial pressure in traumatic brain injury. Cochrane Database Syst Rev. 2006:CD003983.

63. Lazaridis C, Czosnyka M. Cerebral blood flow, brain tissue oxygen, and metabolic effects of decompressive craniectomy. Neurocrit Care. 2012;16:478-84.

64. Randomized evaluation of surgery with craniectomy for uncontrollable elevation of intra-cranial pressure (RESCUE-ICP). http://www.rescueicp.com/frameset4.html. Accessed 15 Dec 2012. 